\title{
Transcranial direct current stimulation (tDCS) for fatigue in multiple sclerosis
}

\author{
Roberta Ferrucci ${ }^{\mathrm{a}}$, Maurizio Vergari ${ }^{\mathrm{a}}$, Filippo Cogiamanian ${ }^{\mathrm{a}}$, Tommaso Bocci ${ }^{\mathrm{b}, \mathrm{c}}$, Matteo Ciocca $^{\mathrm{a}, \mathrm{d}}$, \\ Emanuele Tomasini ${ }^{\mathrm{a}}$, Milena De Riz ${ }^{\mathrm{a}}$, Elio Scarpini ${ }^{\mathrm{a}, \mathrm{d}}$ and Alberto Priori ${ }^{\mathrm{a}, \mathrm{d}, *}$ \\ ${ }^{a}$ Fondazione IRCCS Ca' Granda, Ospedale Maggiore Policlinico, Milano, Italy \\ ${ }^{\mathrm{b}}$ Dipartimento di Neuroscienze, Unità di Neurologia, Università degli Studi di Pisa, Pisa, Italy \\ ${ }^{\mathrm{c}}$ Dipartimento di Neuroscienze, Neurologia e Neurofisiologia Clinica, Università degli Studi di Siena, \\ Siena, Italy \\ ${ }^{\mathrm{d}}$ Dipartimento di Fisiopatologia Medico-Chirurgica e dei Trapianti, Università degli Studi di Milano, \\ Milano, Italy
}

\begin{abstract}
.
BACKGROUND: The debilitating fatigue that patients with multiple sclerosis (MS) commonly experience during day-to-day living activities responds poorly to current therapeutic options. Direct currents (DC) delivered through the scalp (transcranial DC stimulation or tDCS) at weak intensities induce changes in motor cortical excitability that persist for almost an hour after current offset and depend on current polarity. tDCS successfully modulates cortical excitability in various clinical disorders but no information is available for MS related fatigue.

OBJECTIVE: In this study we aimed to assess fatigue symptom after five consecutive sessions of anodal tDCS applied over the motor cortex in patients with MS.

METHODS: We enrolled 25 patients with MS all of whom experienced fatigue. We delivered anodal and sham tDCS in random order in two separate experimental sessions at least 1 month apart. The stimulating current was delivered for 15 minutes once a day for 5 consecutive days. In each session the Fatigue Impact Scale (FIS) and the Back Depression Inventory (BDI) were administered before the treatment (baseline), immediately after treatment on day five (T1), one week (T2) and three weeks (T3) after the last tDCS session.

RESULTS: All patients tolerated tDCS well without adverse events. The fatigue score significantly decreased after anodal tDCS in $65 \%$ of the patients (responders). After patients received tDCS for 5 days their FIS scores improved by about $30 \%$ and the tDCS-induced benefits persisted at $\mathrm{T} 2$ and $\mathrm{T} 3$.

CONCLUSION: Our preliminary findings suggest that anodal tDCS applied over the motor cortex, could improve fatigue in most patients with MS.
\end{abstract}

Keywords: Multiple sclerosis, fatigue, tDCS, treatments, motor cortex

\footnotetext{
*Address for correspondence: Prof. Alberto Priori, MD, PhD, Centro Clinico per la Neurostimolazione, le Neurotecnologie ed i Disordini del Movimento, Fondazione IRCCS Ca' Granda - Ospedale Maggiore Policlinico, via F. Sforza 35, Milano 20122, Italy. Tel.: +39 02 55038671; E-mail: alberto.priori@unimi.it.
}

\section{Introduction}

Patients with multiple sclerosis (MS) commonly complain of fatigue. About $75 \%$ of patients suffer from severe fatigue at some point in the disease course (Bakshi, 2003; Kos et al., 2008). Fatigue severely limits patients' social life and induces marked disability. Currently approved treatments for MS have only a limited 
effect on fatigue (Amato \& Portaccio, 2012) and other newer treatment options often achieve scarce benefit, have high costs and may induce toxicity. Another concern is worsening fatigue related to depression, a frequent comorbidity in MS (Bakshi et al., 2000).

Fatigue related to MS, especially in patients with primary or secondary progressive disease, may manifest at any stage of the illness. Although the pathogenesis involves both central and peripheral abnormalities central changes probably play the major role. Central fatigue probably arises through three main neurophysiological mechanisms: slow conduction along central motor pathways leading to reduced spinal motoneuron recruitment (Gandevia, 2001; Racinais et al., 2007; Sheean et al., 1997), a frequency-dependent conduction block at Ranvier's nodes (Sheean et al., 1997) and impairment of cortical areas involved in motor planning (i.e. the prefrontal cortex) (Leocani et al., 2001; Roelcke et al., 1997).

Current treatment options for MS-related fatigue such as amantadine (Krupp et al., 1995; Pucci et al., 2007), modafinil (Lange et al., 2009; Moller et al., 2011) and serotonin reuptake inhibitors failed to demonstrate significant benefit in controlled trials (Stankoff et al., 2005).

Transcranial direct current stimulation (tDCS) is a simple, inexpensive, non invasive technique that can induce sustained excitability changes in relatively restricted human brain areas (Nitsche \& Paulus, 2000; Priori, 2003). tDCS applied to the motor area for a few minutes in a group of a healthy subjects significantly increased the endurance time for an isometric elbow flexor muscle contraction without inducing adverse effects. Although the real explanation remains unclear, tDCS could benefit muscle fatigue through several mechanisms, for example by facilitating voluntary activation or modulating the brain inhibitory feed-back system. Because central fatigue may depend on frontal area hypoactivation (Roelcke et al., 1997; van Duinen et al., 2007) tDCS could also benefit patients by restoring activation in prefrontal areas. Finally, central fatigue in MS may depend at least in part on a functional disconnection between frontal areas and thalamus (DeLuca \& Nocentini, 2011) probably induced by a dopamine deficiency (Ziv et al., 1998). tDCS could improve the connectivity between these two structures. No studies have investigated whether tDCS improves fatigue in patients with MS, information on its benefits or failure might give patients with MS a new treatment option.

We designed this study to assess whether tDCS over the motor area in the cerebral cortex influenced the symptom fatigue in MS. To do so, in a group of 25 patients we stimulated the cortical motor area for five days with anodal tDCS delivered for 15 minutes at $1.5 \mathrm{~mA}$ or sham tDCS and evaluated fatigue symptoms with Fatigue Impact Scale (FIS) before and for 3 weeks after treatment ended.

\section{Methods}

\subsection{Patients}

We selected 25 patients (18 women and 7 men; age range 23-70 years) with MS diagnosed according to McDonald's criteria (McDonald et al., 2001): 22 had relapsing-remitting and 3 patients secondary progressive MS (Table 1). To be enrolled patients had to have experienced chronic fatigue for at least 6 months with a global score on the Modified Fatigue Impact Scale (MFIS) > 45 (Kos et al., 2005; Tellez et al., 2005), and an Expanded Disability Status Scale (EDSS) (Kurtzke, 1983) score ranging from 0 to 6.5. All patients were screened and recruited at the Multiple Sclerosis Center, Fondazione IRCCS Ca' Granda of Milan. Exclusion criteria were relapse or corticosteroid course in the 2 months before tDCS, pregnancy or breastfeeding; patients were also excluded if they had a history of other neurological or psychiatric disorders. Diseasemodifying therapies such as beta interferon, glatiramer acetate, azathioprine, or methotrexate were allowed, but patients had to be receiving a stable dose for at least 2 months. All symptomatic treatment for fatigue was withdrawn at least 14 days before tDCS. Throughout the tDCS study patients continued taking the same medications at the same doses used during the previous 2 months. The study was conducted according to the Declaration of Helsinki and approved by the institutional review board. Patients and their caregivers gave their informed consent before participation.

\subsection{Experimental protocols}

tDCS was bilaterally delivered with a constant direct current stimulator (HDCKit, Newronika, Italy) connected to three sponge electrodes $(5 \times 7 \mathrm{~cm})$, two placed on the scalp over the motor cortex bilaterally (C3 and $\mathrm{C} 4$ scalp position of the international EEG 10/20 system) and one placed over the right deltoid muscle. The stimulating current was an anodal DC at $1.5 \mathrm{~mA}$ intensity delivered for 15 minutes, once a day for 5 consecutive days. tDCS polarity refers to the electrode over 
Table 1

Clinical features in the 23 patients with multiple sclerosis

\begin{tabular}{|c|c|c|c|c|c|}
\hline Subjects & Gender & Age (years) & Duration of illness (years) & EDSS scores & Type \\
\hline $\begin{array}{l}\text { Responders } \\
(n=15)\end{array}$ & 11 Female & $40.3 \pm 2.3^{*}$ & $12.6 \pm 1.4$ & $3 \pm 0.4$ & $\begin{array}{c}13 \text { SM-RR } \\
2 \text { SM-SP }\end{array}$ \\
\hline $\begin{array}{l}\text { Non-responders } \\
(n=8)\end{array}$ & 5 Female & $52.5 \pm 4.1^{*}$ & $14.4 \pm 5$ & $3.8 \pm 0.7$ & $\begin{array}{l}6 \text { SM-RR } \\
2 \text { SM-SP }\end{array}$ \\
\hline
\end{tabular}

F: female; M: male; EDSS: Expanded Disability Status Scale; SM-SP: secondary progressive; SM-RR: relapsing remitting; ${ }^{*}$ significant difference $p<0.05$. All values are expressed as means \pm SEM.

Table 2

Results for the Fatigue Impact Scale (FIS) assessed before and at three timepoints during a 3-week follow-up after the 23 patients received anodal and sham transcranial direct current stimulation (tDCS)

\begin{tabular}{|c|c|c|c|c|c|c|c|c|}
\hline & \multicolumn{4}{|c|}{ ANODAL } & \multicolumn{4}{|c|}{+} \\
\hline & T0 & $\mathrm{T} 1$ & $\mathrm{~T} 2$ & T3 & $\mathrm{T} 0$ & T1 & $\mathrm{T} 2$ & T3 \\
\hline espon & $59.5 \pm 7.1$ & $38.5 \pm 4.1$ & $40.9 \pm 5.2$ & $39.9 \pm 5.2$ & $49.8 \pm 7$ & $43.1 \pm 5.4$ & $40.9 \pm 7$ & $39.3 \pm 6.5$ \\
\hline Non-responders & $58.5 \pm 10.7$ & $61 \pm 10.7$ & $46.1 \pm 12.6$ & $49 \pm 12.8$ & $61 \pm 11.4$ & $52.5 \pm 12.8$ & $46.3 \pm 13.6$ & $51.8 \pm 13.3$ \\
\hline
\end{tabular}

T0: baseline; T1: immediately after treatment on day five; T2: one week after the end of treatment; T3: 3 weeks after the last tDCS session. All values are expressed as means \pm SEM.

the scalp. For placebo DC, electrodes were placed as for real stimulation but the stimulator was turned off after $10 \mathrm{~s}$. The subjects therefore felt the initial itching sensation when stimulation began but thereafter received no current.

Anodal and sham (i.e. placebo) tDCS were tested in random order in two separate experimental sessions held at least 1 month apart. The patients and the examiner who did the ratings were blind to the type of tDCS delivered in each session. The person who applied tDCS was aware of its polarity. In each session, all patients completed the following assessment scales before the treatment (baseline), immediately after treatment on day five (T1), one week (T2) and three weeks (T3) after the last tDCS session at the same time of day.

\subsection{Scales}

\subsubsection{Fatigue Impact Scale (FIS)}

Fatigue was evaluated using the Italian version of the Fatigue Impact Scale (FIS) (Fisk et al., 1994). The FIS consists of 40 items including three subscales: cognitive, physical and psychosocial functioning. Each item was scored on a five-point scale from 0 (no fatigue) to 4 (severe fatigue).

\subsubsection{Back Depression Inventory (BDI)}

Mood was evaluated with the 21-item Back Depression Inventory (BDI) (Beck et al., 1961). The BDI is a 21-question multiple-choice self-report inventory that consists of items relating to depressive symptoms such as hopelessness and irritability, cognitive symp- toms such as guilt or feelings of being punished as well as physical symptoms such as fatigue, weight loss and lack of interest in sex.

\subsection{Data analysis}

We collected the data for demographic characteristics (age, duration of disease and severity of the disease as measured by the EDSS). Wilcoxon test was used to compare baseline performance. The Friedman test was used to compare all scale scores (FIS and BDI) at baseline and after real or sham tDCS [stimulation: Anodal-Cathodal; time: Baseline-T1] and to compare the effect over time for each stimulation [time: baseline-T1-T2-T3]. Dunn's multiple comparison test was used for post hoc analysis $(p<0.05)$. Patients were further sub-grouped: patients with positive responses after anodal tDCS were defined as 'responders' and those whose responses remained unchanged after anodal tDCS were defined as 'nonresponders'. We used the Spearman test to evaluate a possible correlation between tDCS-induced changes in fatigue with patients' demographic characteristics. Unless otherwise indicated all values are expressed a means \pm SEM. $P$ values $<0.05$ were considered to indicate statistical significance.

\section{Results}

During the study two patients experienced a skin reaction and decided to withdraw. Both adverse 
reactions healed spontaneously within a few weeks. These two patients were considered as drop-outs. None of the other subjects reported adverse reactions after real or placebo tDCS.

When we checked comparability by analyzing our main outcome measure, our patients' performance on the FIS, we found no significant baseline differences between individual patients $(p=0.3)$. Significant correlations were found between fatigue improvement as indexed by FIS and age $(p=0.02)$, but not with duration of disease $(p=0.9)$ and severity of MS $(p=0.3)$. FIS total scores improved more in younger than in older patients.

A preliminary data analysis showed that overall fatigue assessed at $\mathrm{T} 1$ improved significantly after anodal tDCS (T0 vs. T1, $p=0.02$ ) but not after sham tDCS (T0 vs. T1, $p=0.2$ ). Data for individual responses showed that whereas the fatigue score measured at $\mathrm{T} 1$ improved from baseline in 15 patients (about 2/3) and the improvement persisted at the further follow-up visits (responders) at one week and three weeks, in 8 patients it remained unchanged from baseline also at the subsequent follow-up evaluations (non-responders). Further data were therefore analyzed separately for the two groups (Table 2).

The two groups were well-matched for duration and severity of disease $(p>0.05)$, but they differed in age $(p=0.016)$ : responders were younger than nonresponders (the average difference between the two groups was 12.2 years).

\subsection{Responders}

After patients received anodal tDCS for 5 days their fatigue scores on the FIS improved by about $30 \%$ (range 2\%-70\%) and the tDCS-induced benefits persisted about 1 month after treatment ended. At T1, after patients received anodal tDCS their FIS total score significantly decreased whereas after sham-placebo tDCS it remained unchanged $(p=0.009$; post hoc: anodal T0 vs. T1. $p<0.01)$. The anodal tDCS-induced benefit persisted at the follow up-visit $(p=0.08$; T0 vs. T1 $p<0.001$; T0 vs. T2 $p<0.05$; T0 vs. T3 $p<0.05)$ whereas the sham tDCS-induced benefit did not $(p=0.1)$ (Fig. 1). Anodal and sham tDCS left BDI unchanged $(p>0.05)$.

No correlations were found between fatigue improvement as indexed by FIS and age $(p=0.08)$, duration of disease $(p=0.2)$ and severity of MS $(p=0.6)$.

\subsection{Non-responders}

Neither anodal or sham tDCS at the follow-up visit improved 'non-responders' fatigue scores $(p=0.07)$. Anodal and sham tDCS also left the other variables assessed unchanged (BDI, $p>0.05$ ). No correlations were found between fatigue improvement as indexed by FIS and age $(p=0.7)$, duration of disease $(p=0.6)$ and severity of MS $(p=0.8)$.

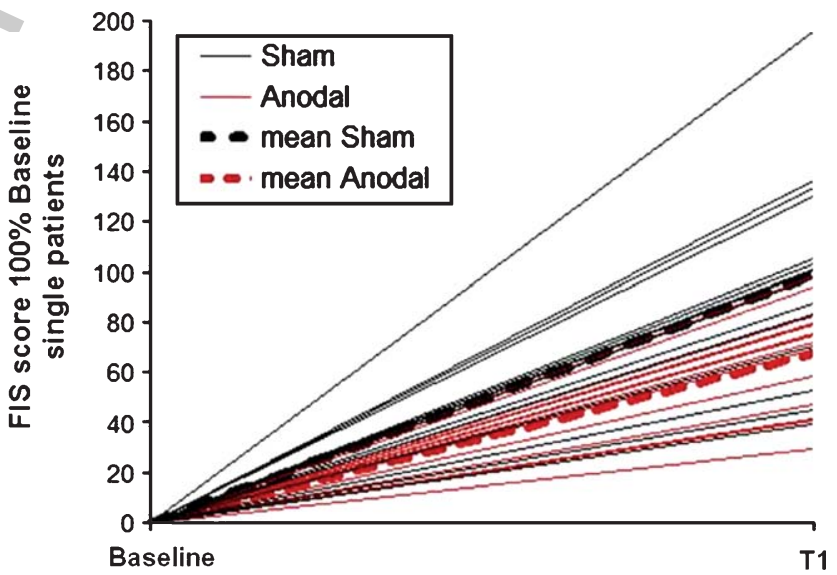

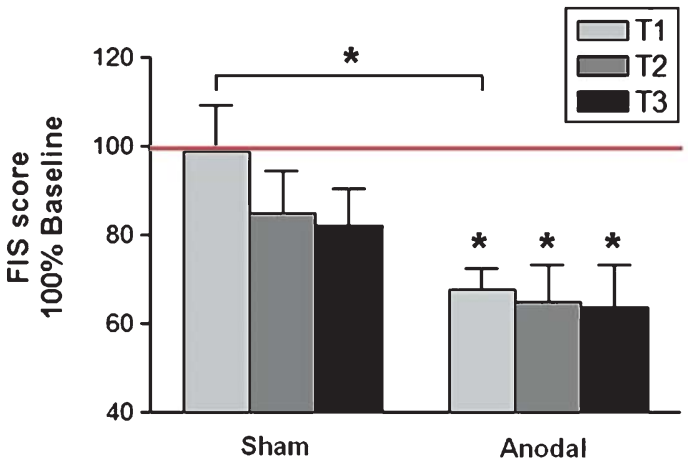

Fig. 1. Effects of transcranial direct current stimulation (tDCS) on Fatigue Impact Scale (FIS) total score in responder patients. (A) The red line indicates baseline values as $100 \%$, and values are calculated as \% of baseline values. T1 (light grey bars) represents the last tDCS session, T2 (dark grey bars) one week and T3 (black bars) three weeks thereafter. Error bars are SEM. Note that after anodal tDCS the FIS total score improved significantly whereas after sham tDCS the scores remained unchanged. ${ }^{*} p<0.05$. (B) Percentage change after anodal (red lines) and sham (black lines) tDCS for the single responder patients. Dot lines represent the mean. Data are variations in FIS score expressed as $\%$ of baseline values $(0$ indicates baseline). 


\section{Discussion}

The main finding is that anodal tDCS delivered for 15 minutes at $1.5 \mathrm{~mA}$ over the motor area in the cerebral cortex improves fatigue symptoms in most patients with MS. Though about $1 / 3$ of patients of patients failed to improve (non-responders), about 2/3 improved significantly and persistently (responders). Sham tDCS left fatigue symptoms almost unchanged over time thus showing that fatigue improvement is a treatmentspecific effect.

Because fatigue improved slightly though nonsignificantly also after sham tDCS we cannot exclude a placebo effect contribution especially after 1 and 3 weeks. We nevertheless consider it unlikely because immediately after tDCS sham tDCS left FIS scores unchanged whereas anodal tDCS improved them significantly.

The first question these findings raise is how anodal tDCS improves fatigue related to MS. Anodal tDCS might have benefitted our responder patients with MS through several mechanisms, including its known antidepressant effect (Brunoni et al., 2012). We can confidently exclude this possibility first because our patients had normal BDI scores for depression and second because even though tDCS improved fatigue it left our patients' depression scales at T2 almost unchanged. Another possibility is that tDCS modulates brain neurochemical content thus improving motor system input-output function. For example, in an experiment combining tDCS with magnetic resonance imaging (MRI) spectroscopy Rango et al. demonstrated that in healthy subjects tDCS significantly and remarkably increased brain myo-inositol (Rango et al., 2008). Brain myo-inositol is an important component in the second messenger system throughout the brain and its increase could improve the neural pathways involved in force generation and endurance. Anodal tDCS might have improved fatigue by decreasing intracortical GABA activity. This reduction could act either to compensate the impaired central drive (Benwell et al., 2007) or to allow subjects to acquire a new motor skill during exercise (Blicher \& Nielsen, 2009). Another possibility is that anodal tDCS increases neuronal and axonal excitability in the human motor cortex (Nitsche et al., 2002; Priori et al., 1998). Because the basic pathophysiological abnormality in MS is a defect in saltatory conduction due to demyelization, a subthreshold electrotonic effect secondary to anodal tDCS could improve axonal conduction along the demyelinated axonal portion. Anodal tDCS might also have transiently facilitated thalamocortical afferents thus enhancing their output to the motor cortex (Leocani et al., 2001). Finally, putative mechanisms linking central fatigue to demyelinization process include the functional uncoupling of regions implicated in movement planning (DeLuca et al., 2009; Sheean et al., 1997), and tDCS could improve fatigue by ameliorating the interconnections between different cortical motor areas (Lang et al., 2004; Williams et al., 2010). Yet a resting state MRI study (Keeser et al., 2011; Pena-Gomez et al., 2012; Polania et al., 2012) showed that tDCS modifies resting-state brain connectivity and could through this mechanism also improve fatigue in MS (Filippi et al., 2012; Rocca et al., 2012). Although the neuro-chemical and neuro-immunological mechanisms underlying fatigue in MS are still largely unknown, given that the constant electric field influences ongoing inflammatory processes (Rueger et al., 2012) tDCS could benefit patients also through this mechanism.

An intriguing issue is why some patients responded whereas other did not. Our study cannot address this unexpected finding but the only difference we found between the two groups was the responders' younger age. This finding could imply that lesions and the pathological abnormalities are less severe in responders than in non-responders thus leaving more space for functional improvement. Against this hypothesis, however, is the observation that no correlation was found between the duration of disease and improvement in responders. Hence an alternative possibility is that the young brain is more susceptible than the older brain to neuroplasticity and hence to the tDCS-induced changes (Boggio et al., 2010; Ross et al., 2011). Finally, studies in depression have shown that genetic polymorphism can influence the response to tDCS (Antal et al., 2010; Malaguti et al., 2011) and other antidepressant treatments (Tsuchimine et al., 2012; Weizman et al., 2012). Along this line of reasoning, the same influence from genetic polymorphism would apply to the tDCS-induced changes in fatigue in patients with MS. Interestingly, the same response heterogeneity has been reported for other treatments used for MS (Kuspinar et al., 2012). Whatever the underlying mechanism, our results are encouraging because the tDCS technique is simple, safe, and well-tolerated and can be used in a large number of patients.

\section{Acknowledgments}

The study was supported by FISM - Fondazione Italiana Sclerosi Multipla - Cod. 2009/R21. 


\section{Declaration of interest}

Tommaso Bocci reports no financial interests or potential conflicts of interests. Emanuele Tomasini reports no financial interests or potential conflicts of interests. Matteo Ciocca reports no financial interests or potential conflicts of interests. Milena De Riz reports no financial interests or potential conflicts of interests. Elio Scarpini reports no financial interests or potential conflicts of interests. Roberta Ferrucci, Filippo Cogiamanian, Maurizio Vergari and Alberto Priori are stakeholders of Newronika s.r.l., a spin-off company of the Fondazione IRCCS Ca' Granda Ospedale Maggiore Policlinico and of the Università degli Studi di Milano.

\section{References}

Amato, M.P., \& Portaccio, E. (2012). Management options in multiple sclerosis-associated fatigue. Expert Opin Pharmacother, 13(2), 207-216.

Antal, A., Chaieb, L., Moliadze, V., Monte-Silva, K., Poreisz, C., Thirugnanasambandam, N., et al. (2010). Brain-derived neurotrophic factor (BDNF) gene polymorphisms shape cortical plasticity in humans. Brain Stimul, 3(4), 230-237.

Bakshi, R. (2003). Fatigue associated with multiple sclerosis: Diagnosis, impact and management. Mult Scler, 9(3), 219-227.

Bakshi, R., Shaikh, Z.A., Miletich, R.S., Czarnecki, D., Dmochowski, J., Henschel, K., et al. (2000). Fatigue in multiple sclerosis and its relationship to depression and neurologic disability. Mult Scler, 6(3), 181-185.

Beck, A.T., Ward, C.H., Mendelson, M., Mock, J., \& Erbaugh, J. (1961). An inventory for measuring depression. Arch Gen Psychiatry, 4, 561-571.

Benwell, N.M., Mastaglia, F.L., \& Thickbroom, G.W. (2007). Differential changes in long-interval intracortical inhibition and silent period duration during fatiguing hand exercise. Exp Brain Res, 179(2), 255-262.

Blicher, J.U., \& Nielsen, J.F. (2009). Cortical and spinal excitability changes after robotic gait training in healthy participants. Neurorehabil Neural Repair, 23(2), 143-149.

Boggio, P.S., Campanha, C., Valasek, C.A., Fecteau, S., PascualLeone, A., \& Fregni, F. (2010). Modulation of decision-making in a gambling task in older adults with transcranial direct current stimulation. Eur J Neurosci, 31(3), 593-597.

Brunoni, A.R., Ferrucci, R., Fregni, F., Boggio, P.S., \& Priori, A. (2012). Transcranial direct current stimulation for the treatment of major depressive disorder: A summary of preclinical, clinical and translational findings. Prog Neuropsychopharmacol Biol Psychiatry.

DeLuca, J., Genova, H.M., Capili, E.J., \& Wylie, G.R. (2009). Functional neuroimaging of fatigue. Phys Med Rehabil Clin N Am, 20(2), 325-337.

DeLuca, J., \& Nocentini, U. (2011). Neuropsychological, medical and rehabilitative management of persons with multiple sclerosis. NeuroRehabilitation, 29(3), 197-219.
Filippi, M., Agosta, F., Spinelli, E.G., \& Rocca, M.A. (2012). Imaging resting state brain function in multiple sclerosis. J Neurol, 260(7), 1709-1713.

Fisk, J.D., Ritvo, P.G., Ross, L., Haase, D.A., Marrie, T.J., \& Schlech, W.F. (1994). Measuring the functional impact of fatigue: Initial validation of the fatigue impact scale. Clin Infect Dis, 18 (Suppl 1), S79-83.

Gandevia, S.C. (2001). Spinal and supraspinal factors in human muscle fatigue. Physiol Rev, 81(4), 1725-1789.

Keeser, D., Meindl, T., Bor, J., Palm, U., Pogarell, O., Mulert, C., et al. (2011). Prefrontal transcranial direct current stimulation changes connectivity of resting-state networks during fMRI. J Neurosci, 31(43), 15284-15293.

Kos, D., Kerckhofs, E., Carrea, I., Verza, R., Ramos, M., \& Jansa, J. (2005). Evaluation of the Modified Fatigue Impact Scale in four different European countries. Mult Scler, 11(1), 76-80.

Kos, D., Kerckhofs, E., Nagels, G., D’Hooghe M, B., \& Ilsbroukx, S. (2008). Origin of fatigue in multiple sclerosis: Review of the literature. Neurorehabil Neural Repair, 22(1), 91-100.

Krupp, L.B., Coyle, P.K., Doscher, C., Miller, A., Cross, A.H., Jandorf, L., et al. (1995). Fatigue therapy in multiple sclerosis: Results of a double-blind, randomized, parallel trial of amantadine, pemoline, and placebo. Neurology, 45(11), 1956-1961.

Kurtzke, J.F. (1983). Rating neurologic impairment in multiple sclerosis: An expanded disability status scale (EDSS). Neurology, 33(11), 1444-1452.

Kuspinar, A., Rodriguez, A.M., \& Mayo, N.E. (2012). The effects of clinical interventions on health-related quality of life in multiple sclerosis: A meta-analysis. Mult Scler, 18(12), 1686-1704.

Lang, N., Nitsche, M.A., Paulus, W., Rothwell, J.C., \& Lemon, R.N. (2004). Effects of transcranial direct current stimulation over the human motor cortex on corticospinal and transcallosal excitability. Exp Brain Res, 156(4), 439-443.

Lange, R., Volkmer, M., Heesen, C., \& Liepert, J. (2009). Modafinil effects in multiple sclerosis patients with fatigue. J Neurol, 256(4), 645-650.

Leocani, L., Colombo, B., Magnani, G., Martinelli-Boneschi, F., Cursi, M., Rossi, P., et al. (2001). Fatigue in multiple sclerosis is associated with abnormal cortical activation to voluntary movement-EEG evidence. Neuroimage, $13(6 \mathrm{Pt} 1)$, 11861192.

Malaguti, A., Rossini, D., Lucca, A., Magri, L., Lorenzi, C., Pirovano, A., et al. (2011). Role of COMT, 5-HT(1A), and SERT genetic polymorphisms on antidepressant response to Transcranial Magnetic Stimulation. Depress Anxiety, 28(7), 568-573.

McDonald, W.I., Compston, A., Edan, G., Goodkin, D., Hartung, H.P., Lublin, F.D., et al. (2001). Recommended diagnostic criteria for multiple sclerosis: Guidelines from the International Panel on the diagnosis of multiple sclerosis. Ann Neurol, 50(1), 121127.

Moller, F., Poettgen, J., Broemel, F., Neuhaus, A., Daumer, M., \& Heesen, C. (2011). HAGIL (Hamburg Vigil Study): A randomized placebo-controlled double-blind study with modafinil for treatment of fatigue in patients with multiple sclerosis. Mult Scler, 17(8), 1002-1009.

Nitsche, M.A., Liebetanz, D., Tergau, F., \& Paulus, W. (2002). [Modulation of cortical excitability by transcranial direct current stimulation]. Nervenarzt, 73(4), 332-335.

Nitsche, M.A., \& Paulus, W. (2000). Excitability changes induced in the human motor cortex by weak transcranial direct current stimulation. J Physiol, 527(Pt 3), 633-639. 
Pena-Gomez, C., Sole-Padulles, C., Clemente, I.C., Junque, C., Bargallo, N., Bosch, B., et al. (2012). APOE Status Modulates the Changes in Network Connectivity Induced by Brain Stimulation in Non-Demented Elders. PLoS One, 7(12), e51833.

Polania, R., Paulus, W., \& Nitsche, M.A. (2012). Reorganizing the intrinsic functional architecture of the human primary motor cortex during rest with non-invasive cortical stimulation. PLoS One, 7(1), e30971.

Priori, A. (2003). Brain polarization in humans: A reappraisal of an old tool for prolonged non-invasive modulation of brain excitability. Clin Neurophysiol, 114(4), 589-595.

Priori, A., Berardelli, A., Rona, S., Accornero, N., \& Manfredi, M. (1998). Polarization of the human motor cortex through the scalp. Neuroreport, 9(10), 2257-2260.

Pucci, E., Branas, P., D’Amico, R., Giuliani, G., Solari, A., \& Taus, C. (2007). Amantadine for fatigue in multiple sclerosis. Cochrane Database Syst Rev(1), CD002818.

Racinais, S., Girard, O., Micallef, J.P., \& Perrey, S. (2007). Failed excitability of spinal motoneurons induced by prolonged running exercise. J Neurophysiol, 97(1), 596-603.

Rango, M., Cogiamanian, F., Marceglia, S., Barberis, B., Arighi, A., Biondetti, P., et al. (2008). Myoinositol content in the human brain is modified by transcranial direct current stimulation in a matter of minutes: A 1H-MRS study. Magn Reson Med, 60(4), 782789.

Rocca, M.A., Valsasina, P., Martinelli, V., Misci, P., Falini, A., Comi, G., et al. (2012). Large-scale neuronal network dysfunction in relapsing-remitting multiple sclerosis. Neurology, 79(14), 14491457.

Roelcke, U., Kappos, L., Lechner-Scott, J., Brunnschweiler, H., Huber, S., Ammann, W., et al. (1997). Reduced glucose metabolism in the frontal cortex and basal ganglia of multiple sclerosis patients with fatigue: A 18F-fluorodeoxyglucose positron emission tomography study. Neurology, 48(6), 15661571.

Ross, L.A., McCoy, D., Coslett, H.B., Olson, I.R., \& Wolk, D.A. (2011). Improved proper name recall in aging after electrical stimulation of the anterior temporal lobes. Front Aging Neurosci, $3,16$.

Rueger, M.A., Keuters, M.H., Walberer, M., Braun, R., Klein, R., Sparing, R., et al. (2012). Multi-session transcranial direct current stimulation (tDCS) elicits inflammatory and regenerative processes in the rat brain. Plos One, 7(8), e43776.

Sheean, G.L., Murray, N.M., Rothwell, J.C., Miller, D.H., \& Thompson, A.J. (1997). An electrophysiological study of the mechanism of fatigue in multiple sclerosis. Brain, 120 (Pt 2), 299-315.

Stankoff, B., Waubant, E., Confavreux, C., Edan, G., Debouverie, M., Rumbach, L., et al. (2005). Modafinil for fatigue in MS: A randomized placebo-controlled double-blind study. Neurology, 64(7), 1139-1143.

Tellez, N., Rio, J., Tintore, M., Nos, C., Galan, I., \& Montalban, X. (2005). Does the Modified Fatigue Impact Scale offer a more comprehensive assessment of fatigue in MS? Mult Scler, 11(2), 198-202.

Tsuchimine, S., Yasui-Furukori, N., Nakagami, T., Sato, Y., \& Kaneko, S. (2012). DRD3, but not BDNF, genotype affects treatment response to paroxetine in major depressive disorder: A preliminary study. J Clin Psychopharmacol, 32(5), 724-726.

van Duinen, H., Renken, R., Maurits, N., \& Zijdewind, I. (2007). Effects of motor fatigue on human brain activity, an fMRI study. Neuroimage, 35(4), 1438-1449.

Weizman, S., Gonda, X., Dome, P., \& Faludi, G. (2012). Pharmacogenetics of antidepressive drugs: A way towards personalized treatment of major depressive disorder. Neuropsychopharmacol Hung, 14(2), 87-101.

Williams, J.A., Pascual-Leone, A., \& Fregni, F. (2010). Interhemispheric modulation induced by cortical stimulation and motor training. Phys Ther, 90(3), 398-410.

Ziv, I., Avraham, M., Michaelov, Y., Djaldetti, R., Dressler, R., Zoldan, J., et al. (1998). Enhanced fatigue during motor performance in patients with Parkinson's disease. Neurology, 51(6), 1583-1586. 